\title{
Sessile macrophages forming clear endotheliumlike membrane on the inside of successful keratoprosthesis *
}

\author{
J. Reimer Wolter ${ }^{1,2}$ and Roger F. Meyer ${ }^{2}$ \\ Departments of ${ }^{1}$ Ophthalmology and ${ }^{2}$ Pathology, University of Michigan Medical Center, Ann Arbor, MI, 48109, USA
}

\begin{abstract}
Clinical observation and cytological study of a successful "through and through" type of Cordona keratoprosthesis, which was removed along with a corneal button about 20 years after its implantation in an aphakic eye, revealed an acellular epitheliumlike film on its outer surface, firm anchoring of its supporting skirt by stable fibrous connections to the stroma, and a continuous separating membrane composed of a homogeneous proteinaceous film with fibroblastlike cells of macrophage origin on its inner surface. The significance of the successful adaptation of the plastic materials of the prosthesis to the tissues of the cornea and the fluid of the inner eye for the future of tissue engineering is discussed.
\end{abstract}

\section{Introduction}

Prosthokeratoplasty is a surgical procedure using clear synthetic materials to replace the central cornea (Cordona 1962, 1969; Cordona et al. 1962; Rizzuti 1983). At present it is used in desperate cases, when transplantation of living tissues has failed or would fail. Two basic types of keratoprostheses are available: the "nut and bolt" type with retrocorneal fixation and the "through and through" kind with stromal fixation by a flexible and fenestrated Teflon skirt. The impressive fact that human eyes can, in principle, survive and see with full-thickness keratoprostheses has been clinically demonstrated beyond doubt. In successful cases, the almost miraculous clinical absence of obstructive scarring on the inner surface of these prostheses, where the foreign substance of their optical portion is continuously exposed to the reactive processes of the anterior chamber, has greatly surprised most morphologically experienced ophthalmologists.

To report the existence of a well-developed and optically clear separating membrane of macrophage origin on the inner surface of a "through and through" type of keratoprosthesis removed 20 years after its implantation is the main purpose of the present paper.

\section{Case report}

At the age of 46 years, this male patient was first seen in our ophthalmology department in January 1954 with a

* Supported by The Research To Prevent Blindness, Inc., New York, NY; Presented on May 22, 1984, at the 120th Meeting of the American Ophthalmological Society in Dorado, Puerto Rico vision of 20/20 in the right eye and 20/30 in the left. Astigmatism was the explanation for the slightly reduced vision in the left eye which also exhibited iris heterochromia. The patient was seen again in January 1959, and at that time his vision could be improved to 20/20 in the right eye and $20 / 25$ in the left. Early cataractous changes were recognized when the patient came back in October 1961. Increasing lens opacities resulted in vision reduction to $20 / 40$ in the right eye and $20 / 80$ in the left by 1 November 1962 . Intracapsular cataract extraction with peripheral iridectomy was done in the left eye without complications 11 days later. Delivery of the lens was described as difficult by the surgeon. Vision in the eye operated upon did not improve immediately after surgery. Corneal edema developed and bullous keratopathy was recognized within a few months after the cataract operation. However, a vision of 20/30-4 was obtained on March 7, 1963, for example, when the cornea was temporarily clear. An intraocular pressure of $25.8 \mathrm{~mm} \mathrm{Hg}$ was observed in the left eye at that time and treatment with $2 \%$ pilocarpine eye drops was started.

Early in 1964 the patient went to South America, saw an ophthalmologist, and was operated upon. A keratoprosthesis was placed in the left eye. The patient came back to the Ann Arbor Eye Department for follow-up. His left eye, with the keratoprothesis, was red and painful; intraocular pressure was $51 \mathrm{~mm} \mathrm{Hg}$. Diamox systemically and pilocarpine drops were effective and brought the pressure down to $28 \mathrm{~mm} \mathrm{Hg}$. At first, vision was finger counting at 10 feet. The situation improved with time and on August 31,1966 , for example, central vision in the left eye had improved to $20 / 40$, but visual field testing revealed much peripheral field constriction. In comparison with the normal eye on the right side, the left eye with the keratoprosthesis was found not to be very useful for binocular function by the patient. However, the eye remained in its normal position, and the patient enjoyed showing off his eye with the "artificial plastic window" (Fig. 1) and was satisfied. One of the authors had the opportunity of following this patient clinically between 1966 and 1983.

By August 1981, the best corrected central vision in the left eye was $20 / 70$, and the intraocular pressure was within normal limits. Slit-lamp examination, surprisingly, revealed a firm and smooth epitheliumlike layer on the anterior surface of the keratoprosthesis. This was optically clear but did stain diffusely with fluorescein. The cornea surrounding the keratoprosthesis was mildly opaque and exhibited slight superficial neovascularization. The anterior chamber was formed and the vitreous appeared clear. The 
fundus was visible only to the midperiphery due to the tubelike shape of the optical portion of the plastic implant. The optic nerve exhibited definite glaucomatous cupping and some atrophy.

In July 1983, the patient suffered a slight injury to his left eye during garden work. On July 12, 1983, he came to the elinic with a red and painful left eye. The eye was soft to finger palpation. In spite of obvious evidence of corneal rupture next to the keratoprosthesis, vision was still $20 / 400$ in the left eye. Slit-lamp examination revealed loss of epithelial surface and stromal substance around the lower aspect of the keratoprosthesis. Leakage of aqueous humor could be demonstrated in this zone. The patient was admitted and a penetrating corneal transplant was done in the left eye on July 15, 1983. The postoperative course was uncomplicated. The graft healed in very well and remained clear. Only mid-September vision in the left eye had improved to $20 / 50$ and intraocular pressure was $20 \mathrm{~mm} \mathrm{Hg}$. The patient was last seen on August 21, 1984. His eye was comfortable and the vision $20 / 25$ with correction. The corneal transplant was crystal clear, the anterior chamber was deep and quiet, the pupil was round with an open iridectomy superiorly, and no vitreous was present in the anterior chamber. Central corneal thickness was recorded as $0.48 \mathrm{~mm}$. Intraocular pressure was $21 \mathrm{~mm} \mathrm{Hg}$ by Goldmann applanation. The treatment the left eye receives at present is: fluorometholone (FML) drops b.i.d., timolol maleate (Timoptic) $0.5 \%$ drops b.i.d., dipivefrine (Propine $0.1 \%$ ) drops bid, pilocarpine $4 \%$ drops q.i.d., and methazolamide (Neptazane) $25 \mathrm{mg}$ by mouth, b.i.d.

\section{Cytopathological study}

The corneal button with the keratoprosthesis in its center measured $8 \mathrm{~mm}$ in diameter. Transillumination of this button showed the optic portion of the prosthesis to be almost clear, but the pattern of a partly damaged grayish layer on its anterior surface was clearly visible (Fig. 2). The Teflon skirt of its haptics could be demonstrated by transillumination. An air bubble indicated separation between the anterior stroma and the Teflon skirt in the inferior region of the leak (Fig. 2). The corneal button was cut in half along the upper edge of the optics of the prosthesis. Using blunt dissection by spreading a small pair of scissors worked well for separating the prosthesis from the corneal tissues. The so-called lens-implant cytology technique (Wolter 1982a) was used to demonstrate the membranes on the front and back surfaces of the prosthesis.

The front surface of the optical portion of the prosthesis was covered with the remnants of what clinically had been repeatedly observed to be a continuous membrane in this position, before the eye developed its recent leakage. This layer was slightly eosinophilic and resembled, in its nature and composition, evenly distributed condensation of acellular epithelial substance (Figs. 3 and 4). This layer was damaged and partly missing on the specimen, but the remaining portions of it were quite firmly attached to the plastic surface and stayed fixed to it through the staining and the lipid-dissolving dehydration process. Seen at high power, this layer exhibited a subtle pattern that indicated its composition of desquamated and compacted epithelial cell remnants without nuclei (Fig. 4).

The posterior surface of the optic was also covered by a firmly adherent and slightly eosinophilic film. This was optically clear, thin, and of homogeneous composition. This continuous filmlike layer was populated by numerous small cells (Figs. 5-7). In the center, these cells were mostly starshaped, had branching processes, and contained pigment granules in their protoplasm (Fig. 6). In the periphery this membrane exhibited more densely arranged cells of a similar type, but they were of denser arrangement, a more irregular shape, and partly exhibited long fiberlike processes (Figs. 5 and 7). The membranous film with its fibroblastlike cells (sessile macrophages) extended to the border of the posterior optic, where Descement's membrane of the surrounding cornea had been cut. Some cytologically typical, free-moving macrophages were also found on the posterior surface of the membrane (Figs. 6 and 7).

The Teflon skirt did not exhibit any inflammatory or giant cells in all those parts of its surface that had been firmly embedded in the corneal stroma. However, in the area of the recent leakage and separation, several large islands of epithelium were found to have grown down to the anterior surface of the Teflon skirt. These were in firm and direct attachment to the Teflon without any underlying mesodermal structures (Fig. 8).

The corneal button had been cut in half for removal of the keratoprosthesis. The two halves were embedded in paraffin and cut - one-half in cross sections and the other in flat serial sections. As expected, an accumulation of acellular epithelial debris resembling the substance covering the anterior surface of the keratoprosthesis was found at the junction between the anterior surface of the prosthesis and the adjacent epithelial surface of the cornea (Fig. 9). This debris had the same composition as the filmlike deposition on the outer surface of the prosthesis described above. Epithelial downgrowth to the level of the Teflon skirt of the prosthesis was found in the area of the leakage. The corneal stroma was in direct contact with the Teflon material of the skirt everywhere, without exhibiting any significant cellular reaction. The anchoring connections of stromal substance through the holes in the skirt were beautifully demonstrated (Fig. 10). These were ruptured in the process of removing the prosthesis, of course. Descemet's membrane was firmly contained in the stromal scarring next to the posterior rim of the prosthesis (Fig. 9). Some clotted blood with an adjacent large giant cell of the foreign-body type was observed on the posterior aspect of the junction between prosthesis and surrounding cornea next to the area of the leak (Fig. 11). The endothelium on the posterior surface of the ring-shaped corneal button exhibited advanced atrophy (Fig. 10).

\section{Discussion}

It was a remarkable experience to clinically observe the eye of this patient with the keratoprosthesis that had been placed almost 20 years ago and to have a chance to study the reaction on and around the plastic implant histologically and cytologically. For many years there were no complications with the keratoprosthesis - and it is important to emphasize that the eye already had glaucoma before its placement. The keratoprosthesis was probably also not really indicated by most accepted standards, and it did not have much useful visual function. However, it has to be stated that this generally intelligent patient certainly was happy and satisfied with it. 


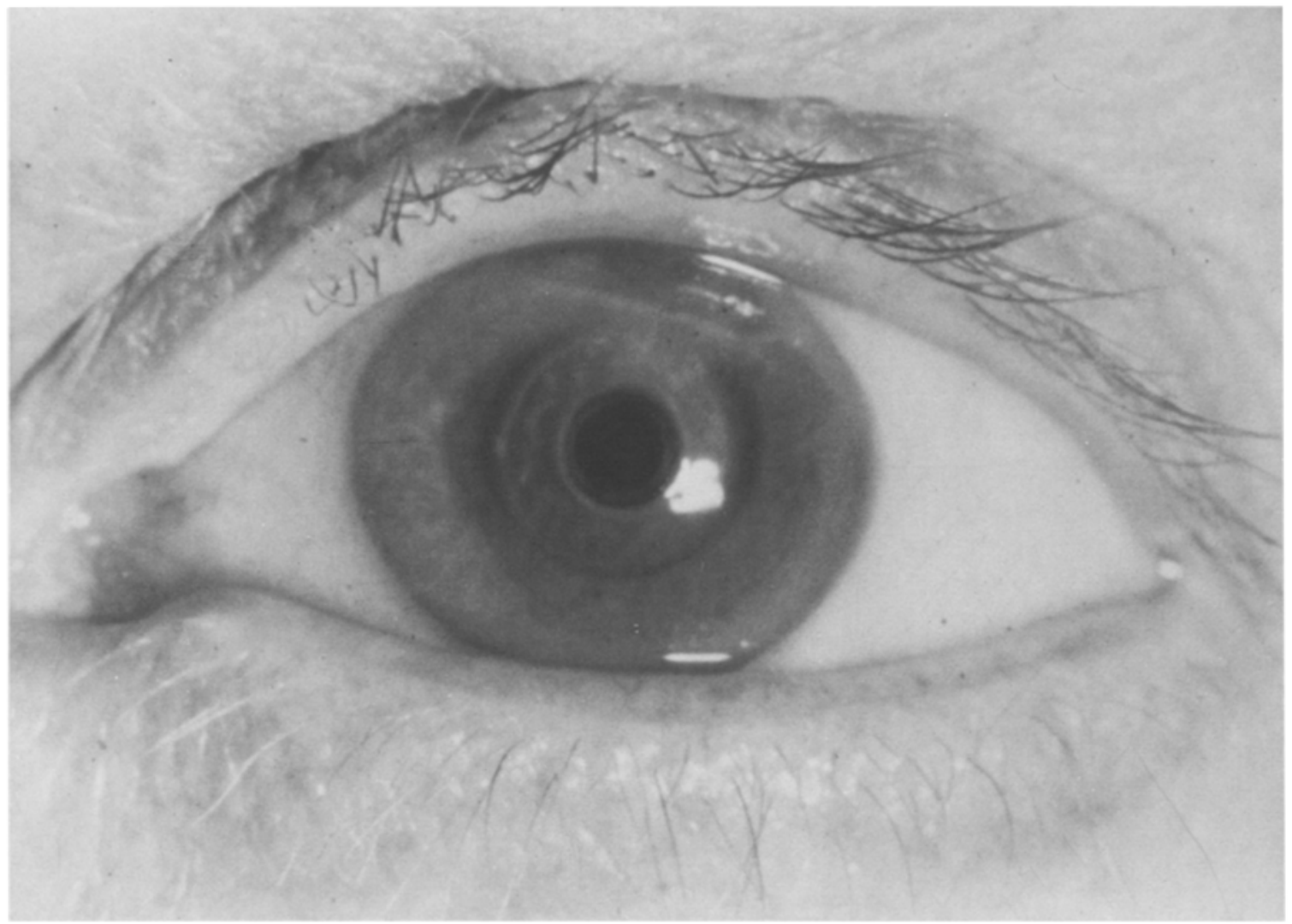

Fig. 1. Clinical view of the left eye with the keratoprosthesis in place. Photograph $\times 4$

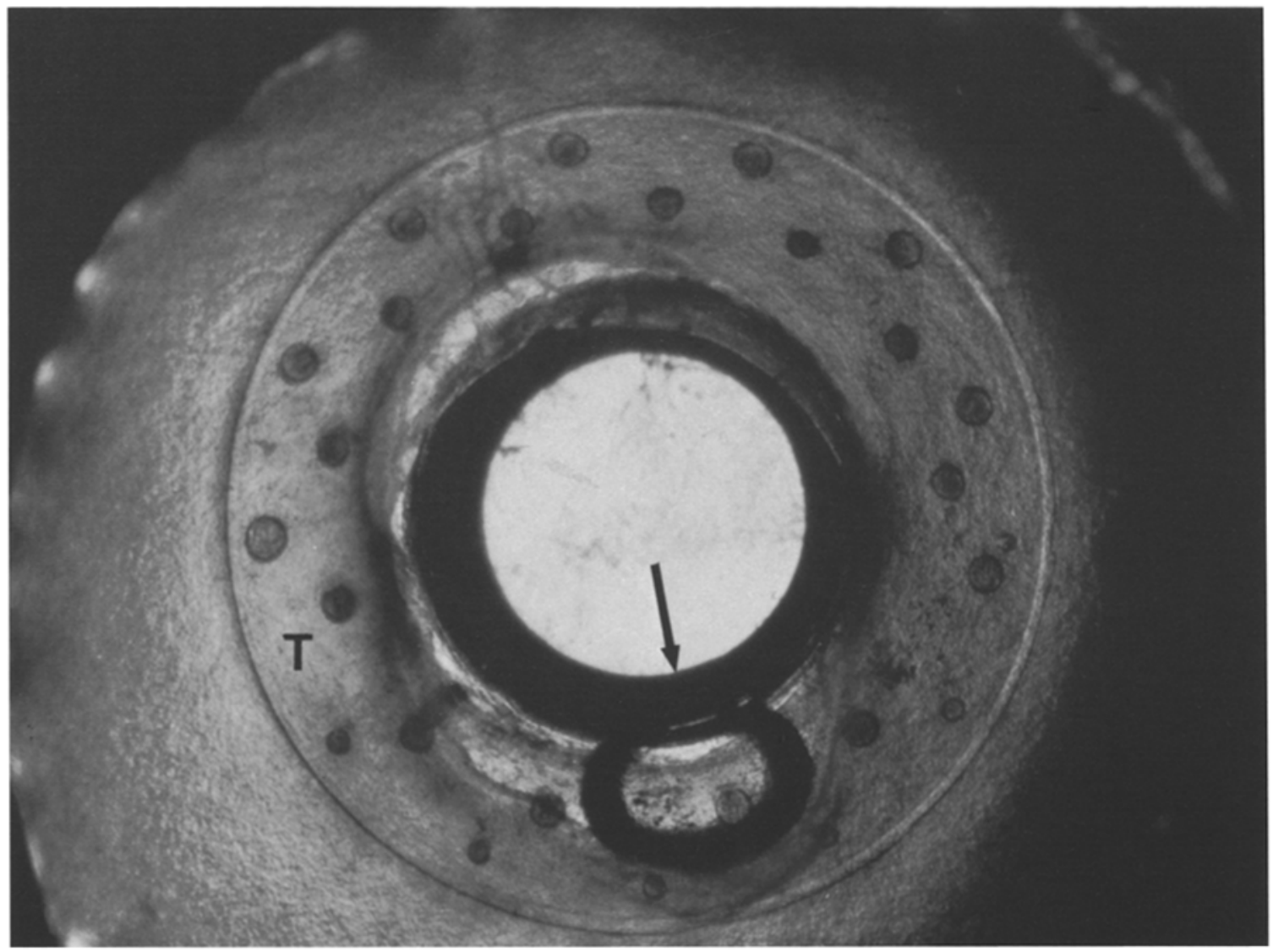

Fig. 2. Transillumination view of the excised corneal button with the keratoprosthesis in the center. The supporting Teflon skirt with its regular perforations $(T)$ is clearly seen. The arrow points to the air bubble in the region of the leak. Photograph $\times 25$ 


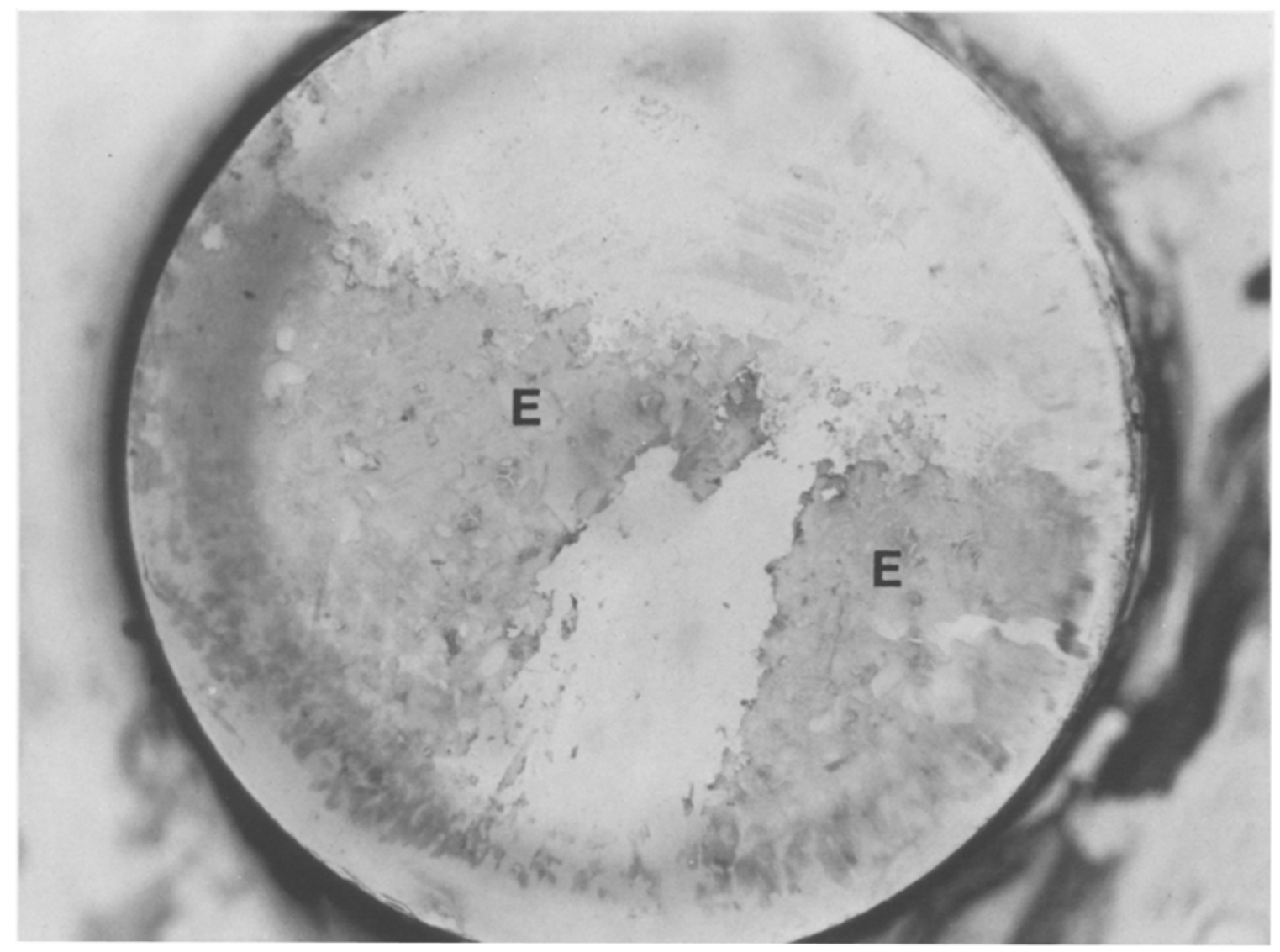

Fig. 3. Front surface of optic portion of keratoprosthesis with remaining parts of epitheliumlike layer $(E)$. Lens-implant cytology technique, $\mathrm{H} \& \mathrm{E}$ stain, photomicrograph $\times 100$

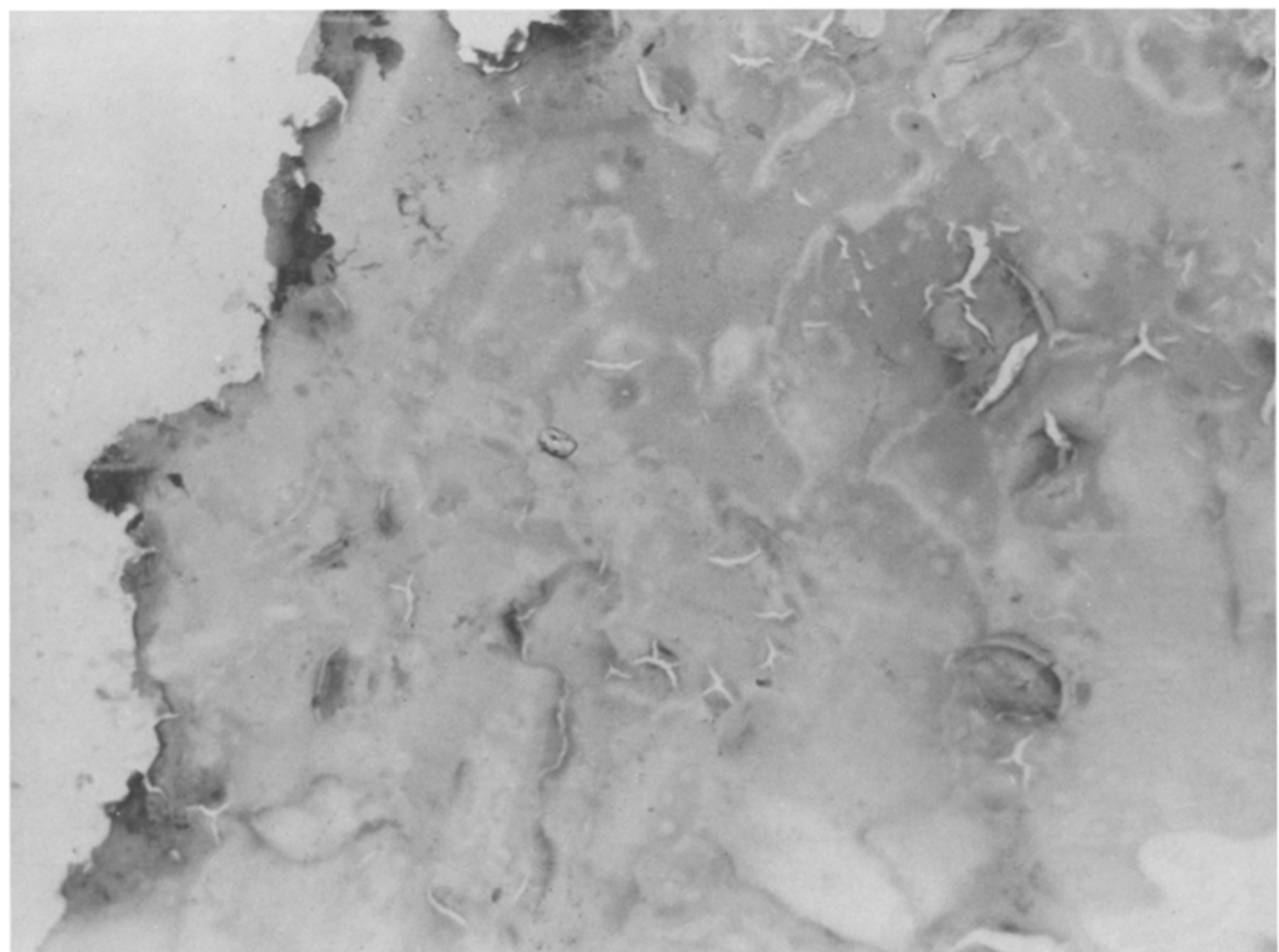

Fig. 4. Higher power view of the epitheliumlike layer on the front surface of the optic portion of the keratoprosthesis with its artificial border to the left. Lens-implant cytology technique, H \& E stain, photomicrograph $\times 300$ 


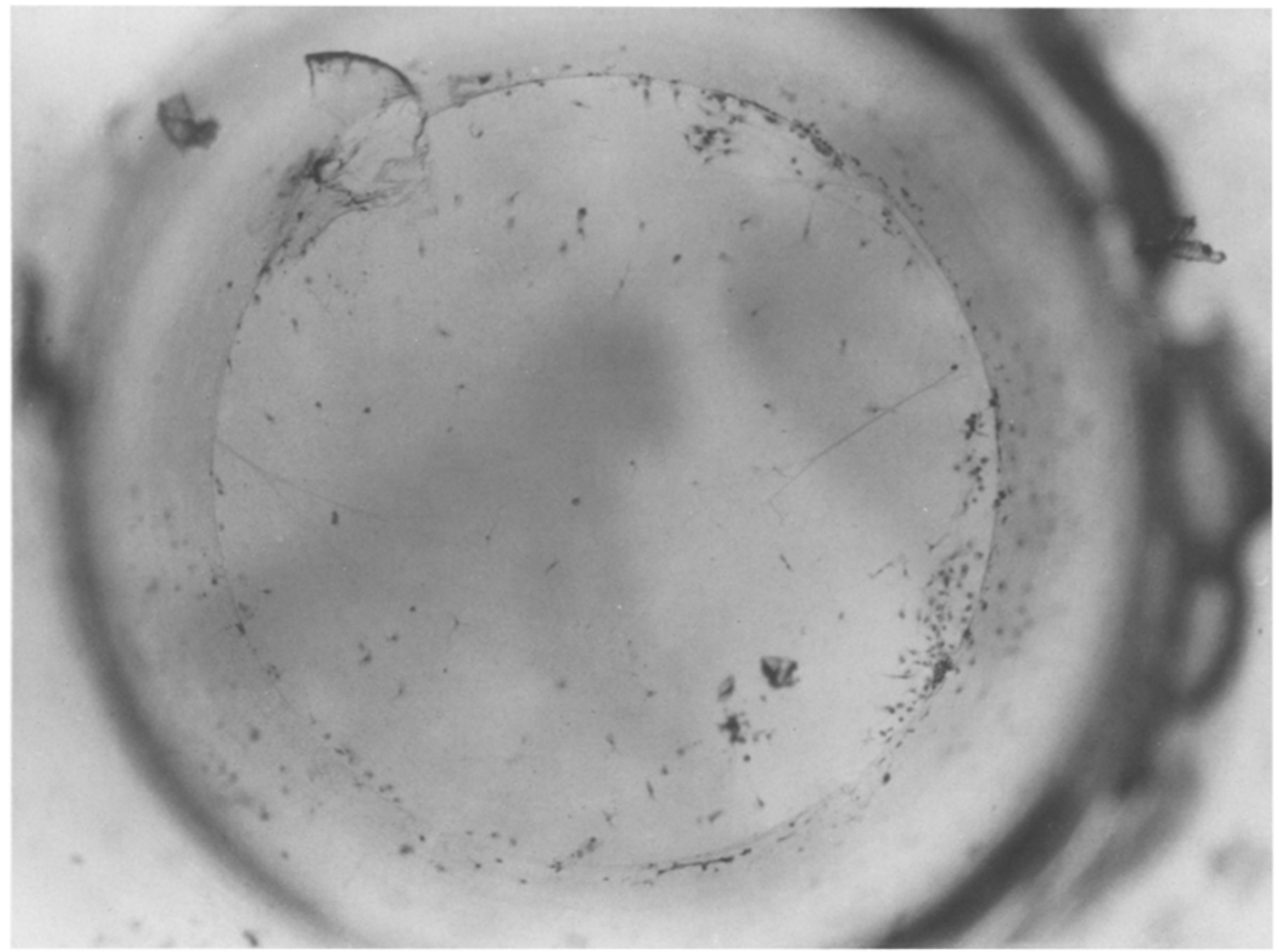

Fig. 5. The eosinophilic film of the posterior surface of the optics of the keratoprosthesis containing numerous fibroblastlike cells. The Teflon rim of the optic portion is clearly visible. Lens-implant cytology technique, H \& E stain, photomicrograph $\times 100$

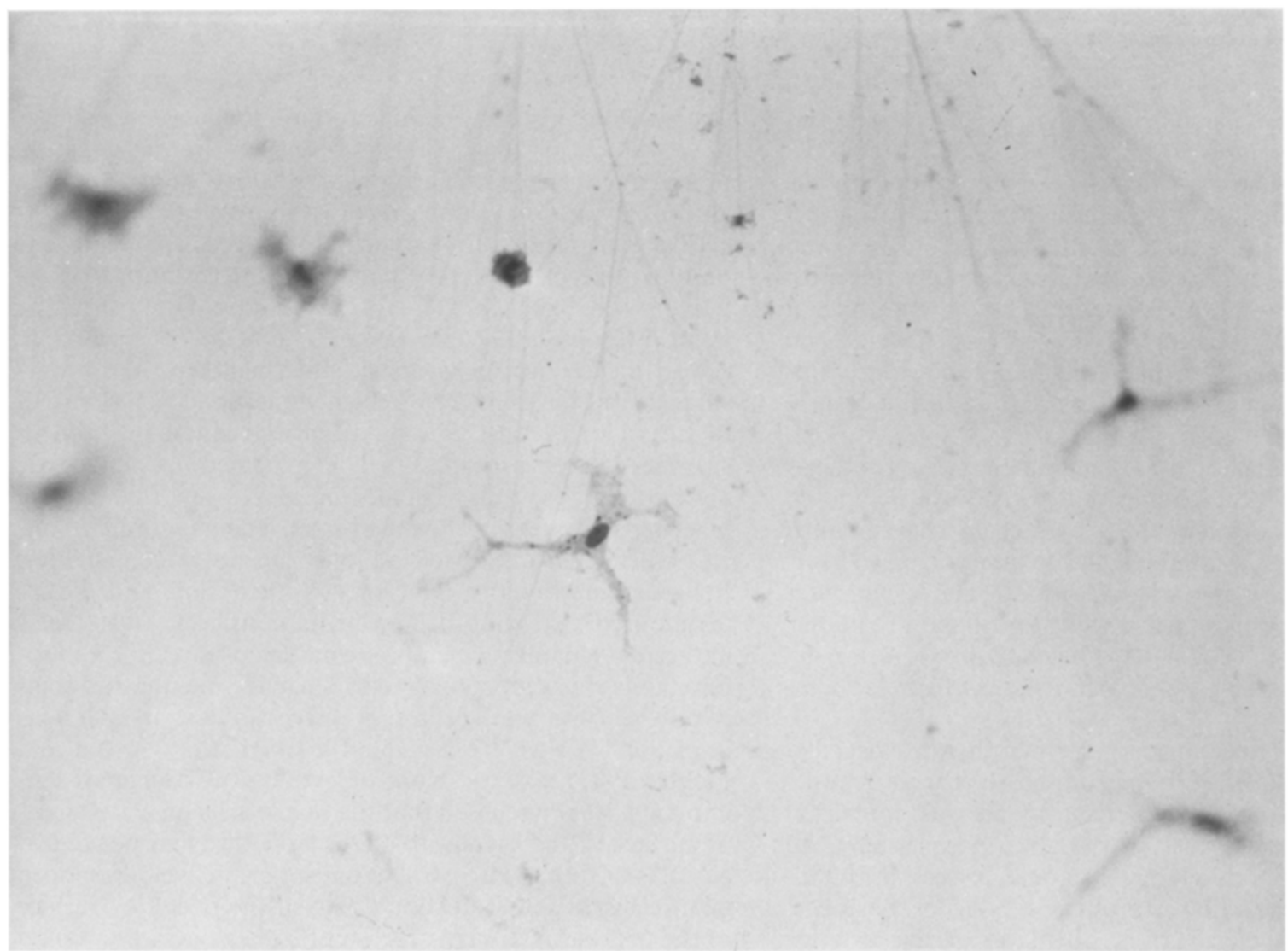

Fig. 6. Higher power view of sessile fibroblastlike cells with branching processes and one round free-moving macrophage (above center) in the central region of the posterior surface of the optics of the keratoprosthesis. Lens-implant cytology technique, $H \& E$ stain, photomicrograph $\times 300$ 


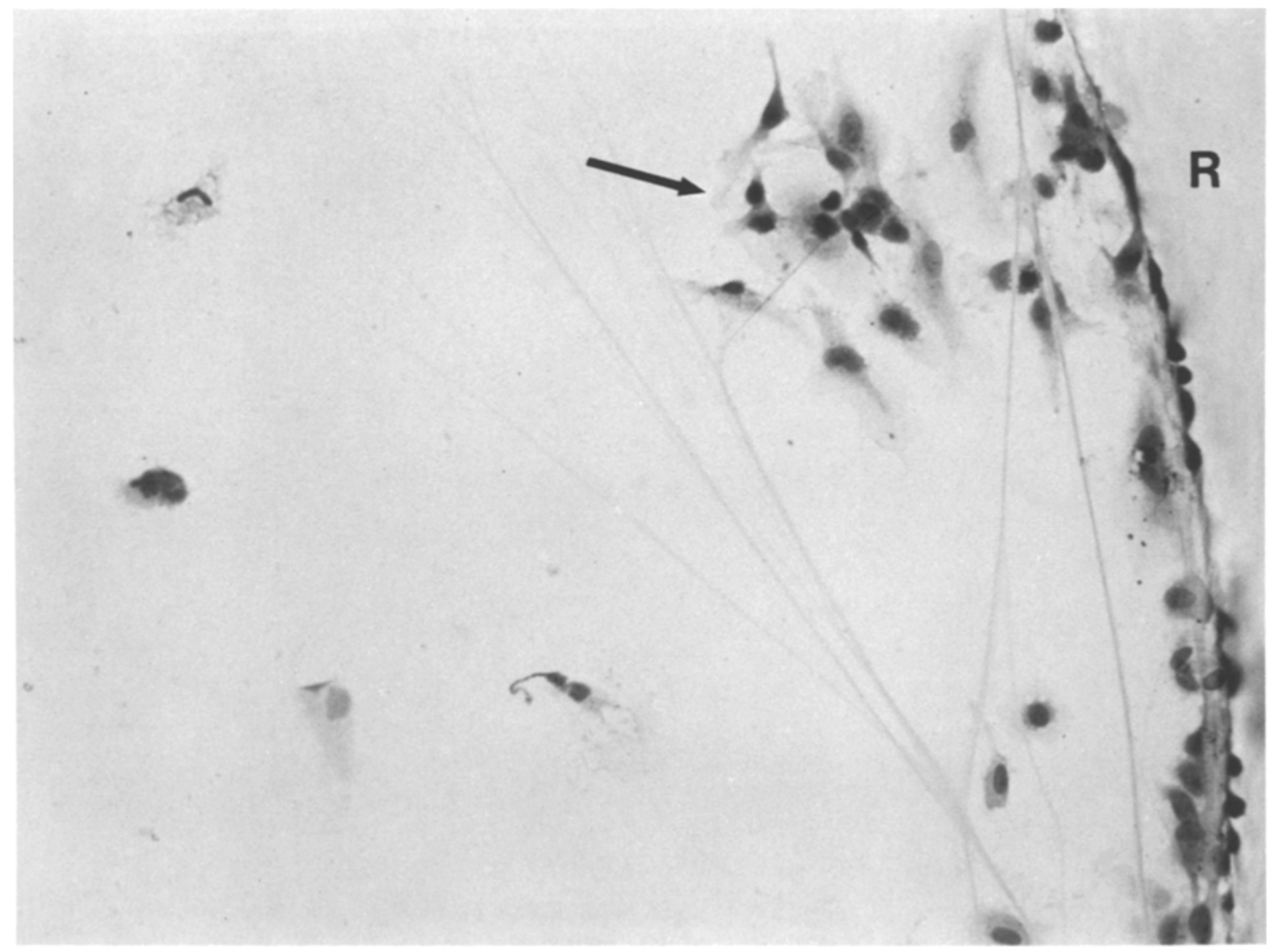

Fig. 7. Marginal fibroblastlike cells with long cellular processes arranged in groups (arrow) in the cosinophilic film on the posterior surface of the optic portion of the keratoprosthesis. The Teflon rim $(R)$ is seen on the right. Single fibroblastlike cells to the left. Lens-implant cytology technique, H \& E stain, photomicrograph $\times 300$

The tissue adjustment around the piece of plastic implanted into the cornea can be subdivided into three separate processes: the adjustment of the front surface of the implant to the epithelial surface, the attachment of its periphery with the Teflon skirt to the corneal stroma, and the formation of a separating membrane on its posterior surface toward the anterior chamber. The outer surface of the optic portion of the keratoprosthesis was clinically and cytologically found to have an epitheliumlike covering. This was smooth, thin, and clear, but in contrast to the corneal epithelium, it readily took up fluorescein stain. Cytologically, this layer was made up of an epitheliumlike proteinaceous substance without living cells, obviously composed of desquamated and compacted epithelial cell remnants. This was rather firmly adherent to the anterior plastic surface and served as a remarkably smooth and clear outer covering for the exposed plastic surface of the prosthesis. In staining poorly cleaned hard contact lenses after extended wear, one of us has seen depositions of similar composition.

The stromal connections to the peripheral aspects of the keratoprosthesis with its perforated skirt were scarlike and fibrous in nature. The fibrous tissue was connected directly to the plastic parts of the skirt. Surprisingly, no separating foreign-body giant cells could be seen at the interface between avascular stroma and plastic substance. Some epithelial downgrowth to the level of the anterior surface of the skirt in the area of leakage was considered to be the result of the recent trauma. The fact that the stromal connections were able to hold the keratoprosthesis in the desired place for almost 20 years without dislocation or leakage in spite of glaucoma with a recorded pressure around $50 \mathrm{~mm} \mathrm{Hg}$ at one time, is truly remarkable.

Demonstration of the optically clear reactive membrane on the inside of the optical portion of the keratoprosthesis, finally, was the high point of this study. The membrane was continuous and composed of a homogeneous proteinaceous film of firm consistency and small bipolar or starshaped fibroblast-like cells (sessile macrophages). Some of these had long processes and virtually all contained pigment granules in their protoplasm. This indicated the phagocytic abilities of these cells. There were also a few free-moving macrophages seen on the membrane. The membrane, thus, was considered quite well established and rather inactive. It had connections to Descement's membrane and the remaining corneal endothelium in its periphery. In its basic nature the separating membrane on the inside of the keratoprosthesis was very similar to the inactive membranes commonly seen on the surface of successful intraocular lens implants (Wolter 1982a, b, 1983a, 1984; Wolter and Lichter 1983). On the basis of cytological studies of lens implants, it is concluded that the sessile cells on the posterior surface of the keratoprosthesis were of macrophage origin. It is of special interest that one large and well-developed giant cell was found at the margin of the posterior surface of the prosthesis near the region of leakage next to a blood clot. This type of giant cell is believed to form by fusion of macrophages and indicates activity (Wolter 1983b), but 


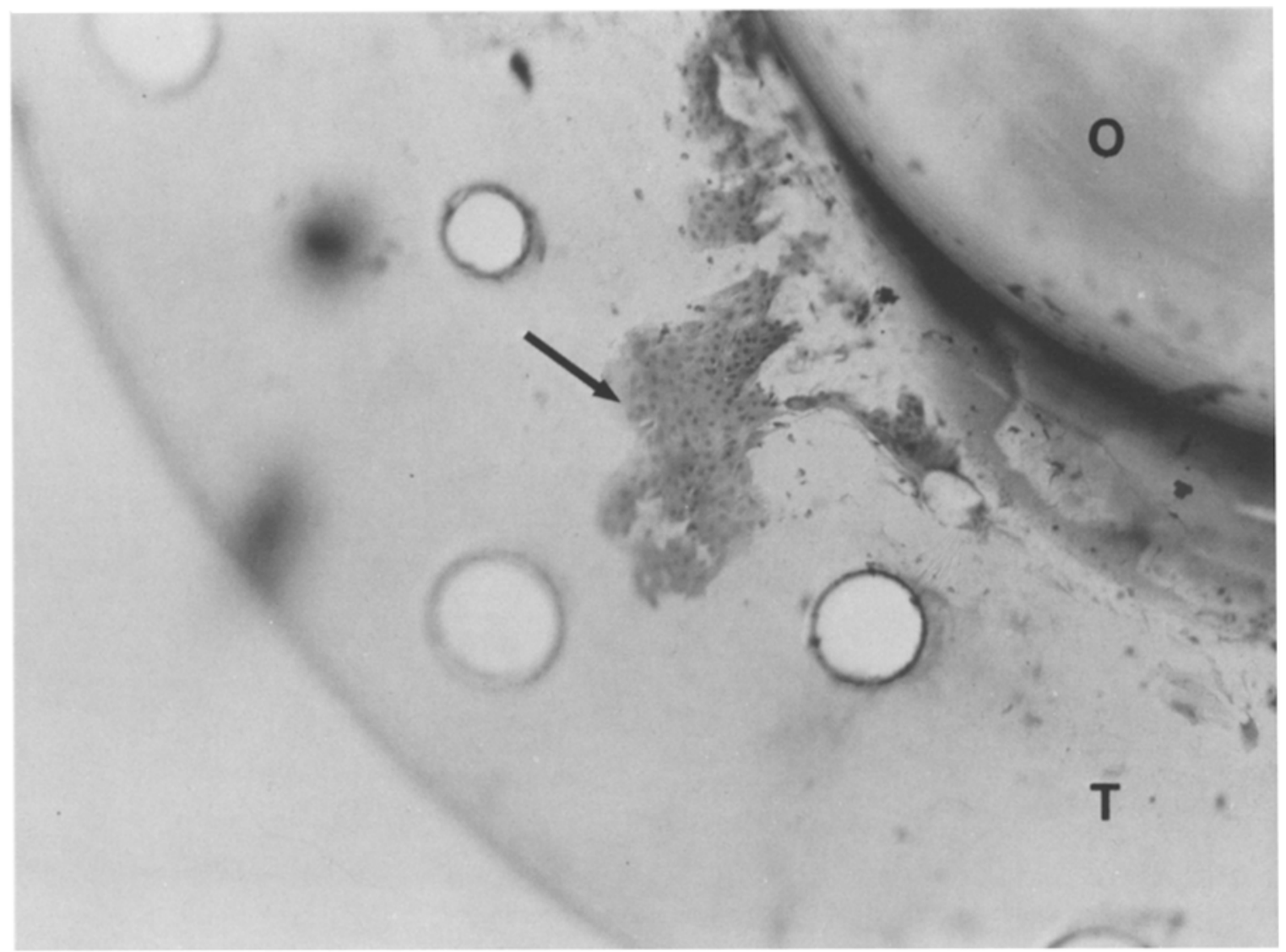

Fig. 8. An island of surface epithelium (arrow) on the anterior aspect of the Teflon skirt in the region of the leak. Optic portion $(O)$ to the right, Teflon skirt $(T)$ has perforations for anchoring in the corneal stroma. Lens-implant cytology technique, H \& $\mathrm{E}$ stain, photomicrograph $\times 100$

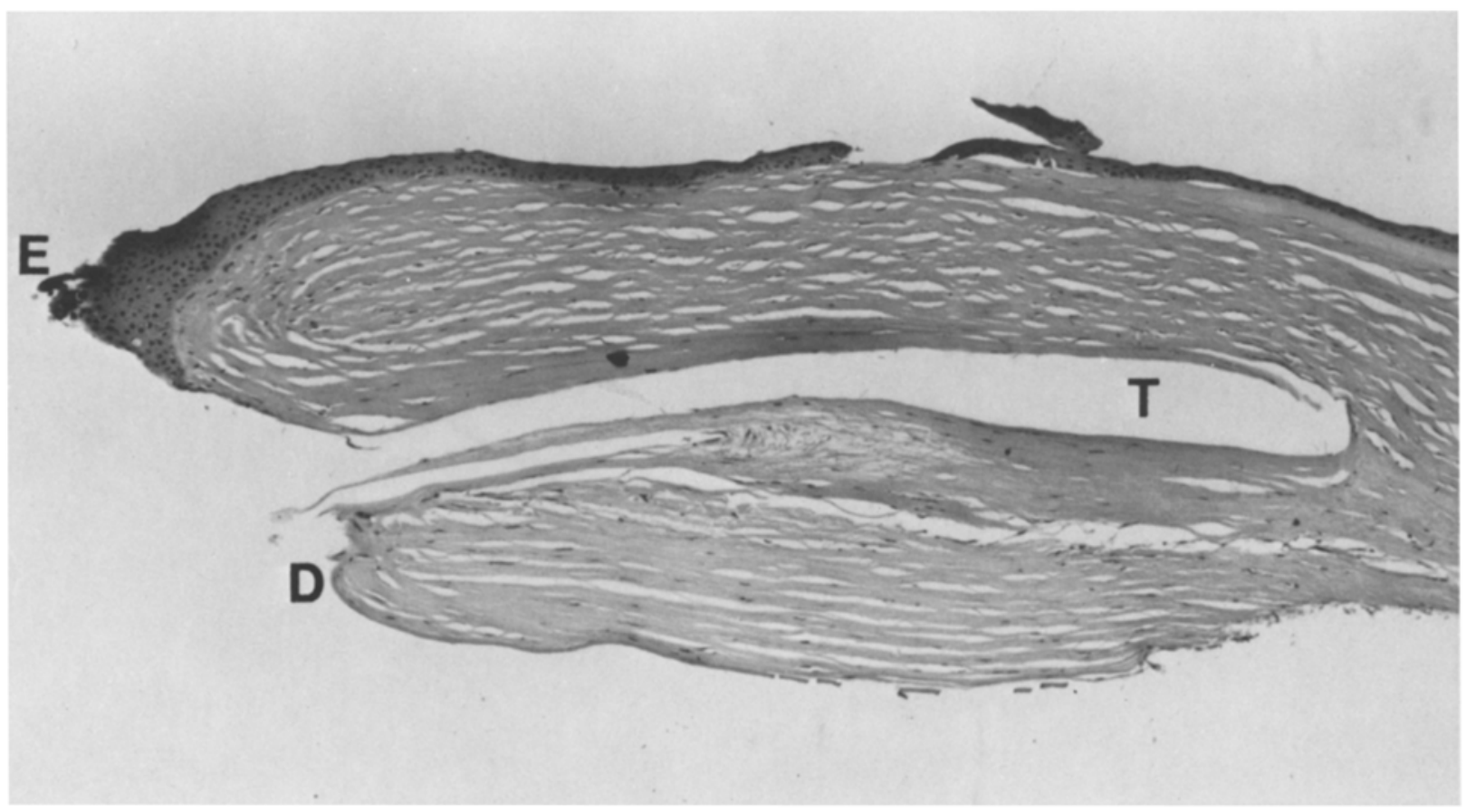

Fig. 9. Cross section of the corneal button shows central aspect with accumulation of acellular epithelial remnants (E) at the limit between living corneal epithelium and anterior surface of kerotoprosthesis. The slit in the middle of the stroma for the supporting Teflon skirt $(T)$ is clearly seen. Descemet's membrane $(D)$ is slightly rolled in and ends next to the posterior rim of the keratoprosthesis. Paraffin section, $\mathrm{H} \& \mathrm{E}$ stein, photomicrograph $\times 100$ 


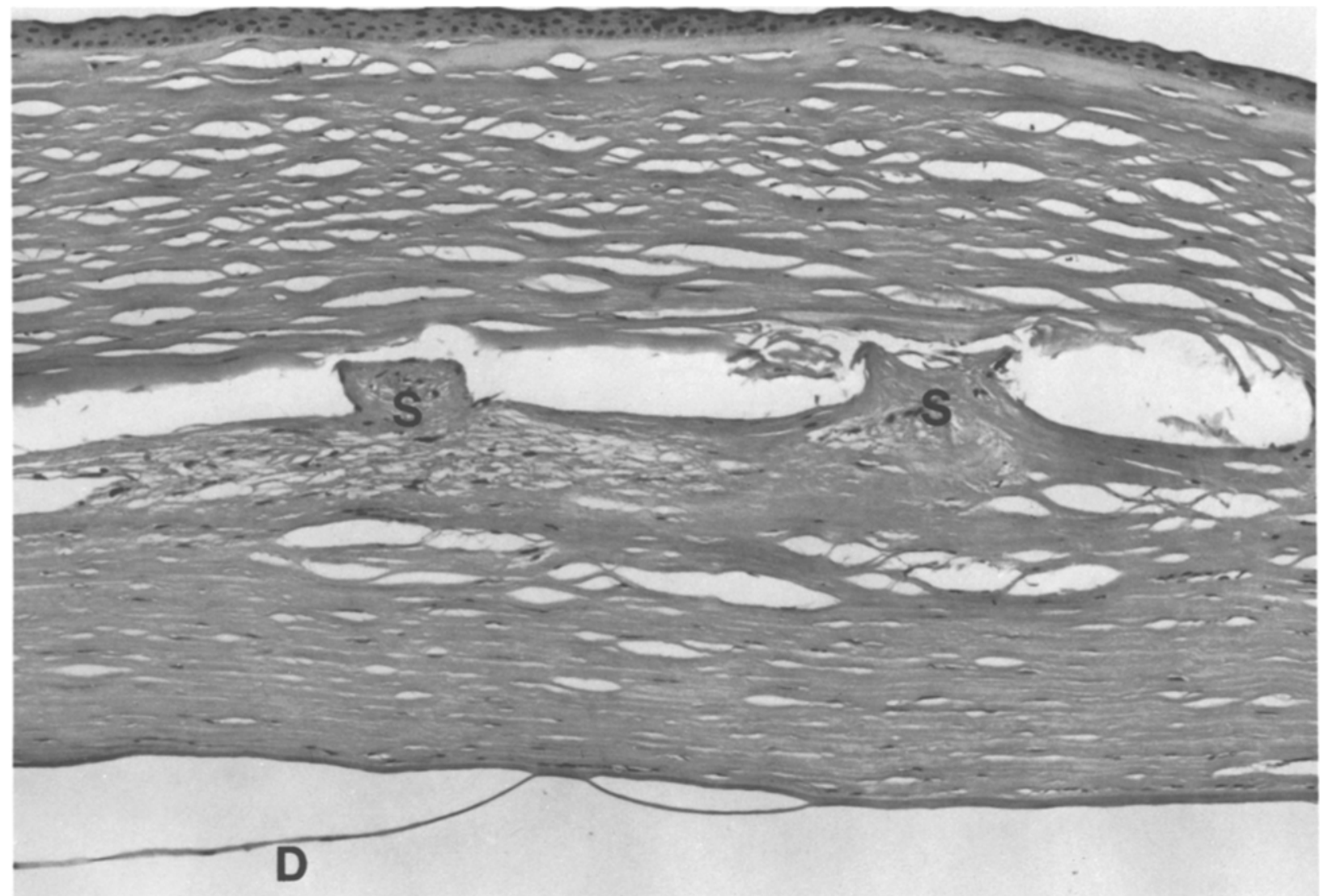

Fig. 10. Portion of the excised cornea with buttonlike scar tissue $(S)$ bridging the space for the Teflon skirt in the region of its anchoring perforations. The stroma is scarred diffusely. The artificially detached endothelium $(D)$ exhibits distinct loss of endothelial cells. Paraffin section, H \& E stain, photomicrograph $\times 150$

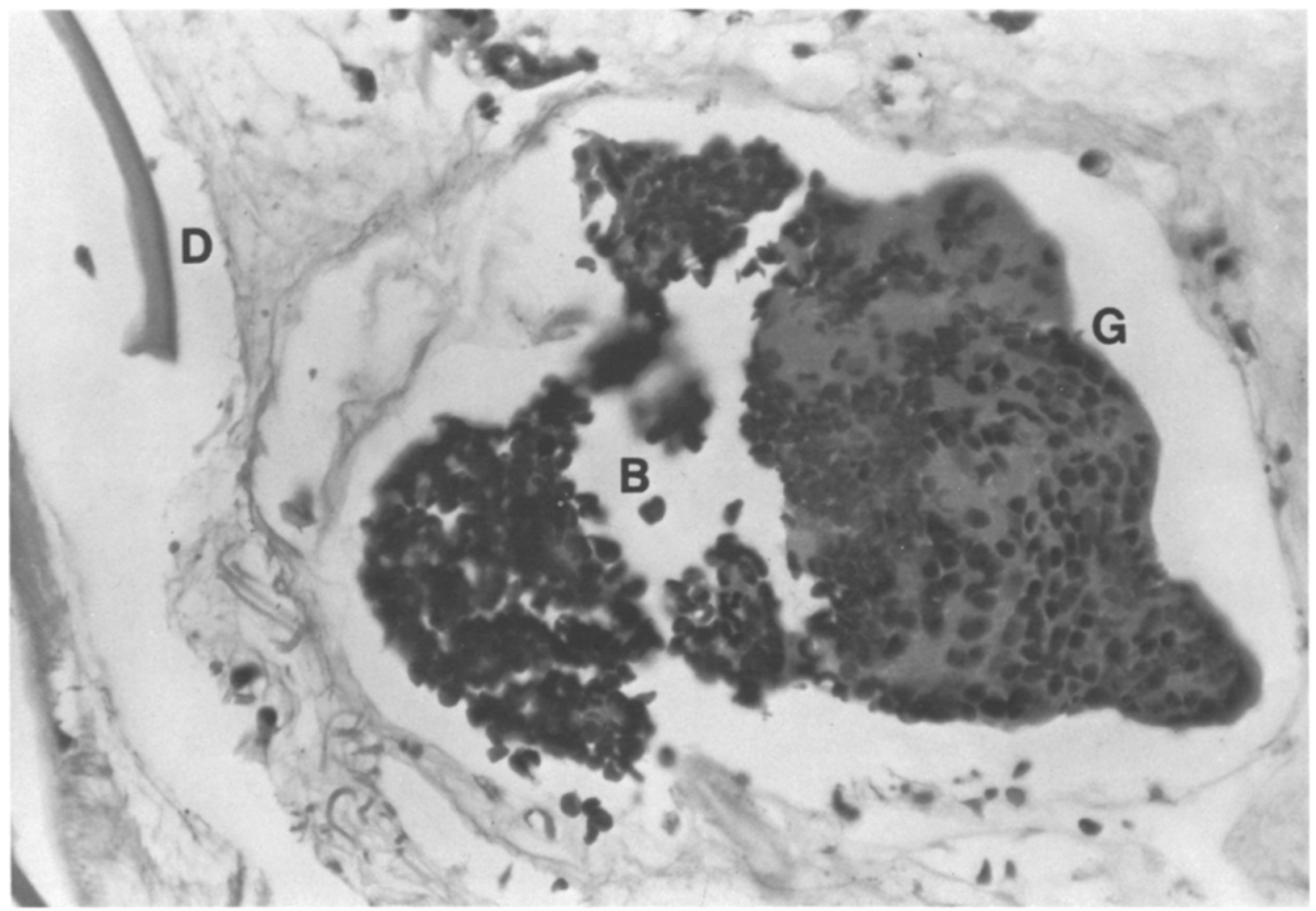

Fig. 11. Multinucleated foreign-body giant cell $(G)$ with about 100 nuclei in the plane of this section next to a blood clot $(B)$ on the posterior aspect of the keratoprosthesis in the region of the recent leak. A portion of Descemet's membrane $(D)$ is also seen. Paraffin section, $\mathrm{H} \& \mathrm{E}$ stain, photomicrograph $\times 300$ 
this could well be a reaction to blood complicating the recent rupture and not a direct reaction to the keratoprosthesis.

The formation of optically obstructive fibrous membranes in back of keratoprostheses has been described as a typical and relatively common complication of prosthokeratoplasty (Barnham and Roper-Hall 1983; Aquavella et al. 1982). The retroprosthetic membrane in the present case, in contrast, is very delicate and optically clear. It is considered a beneficial adaptation process. Green, furthermore, has demonstrated foreign-body giant-cell reaction on the posterior surface of a keratoprosthesis in association with vitritis, retinal phlebitis, cystoid macular edema, and choroiditis (1984). The fact that continuous proteinaceous membranes containing cells of macrophages origin are a typical reaction on the surface of all kinds of foreign substances exposed to the fluids of the anterior ocular segment is supported by the recent observation of the same type of membrane on a piece of glass removed 2 years following perforating injury from the anterior chamber of a child (Wolter and Sugar 1984, in press).

The inner eye functions by transmitting and focusing light from the environment onto the retina. After observing the facts of the present case, one is drawn to the conclusion that the reactive cellular components contribute towards the eye's purpose by attempting to prevent light scattering, even under totally unusual conditions. When a clear pice of plastic was placed in the center of the scarred cornea in the present case, macrophages were mobilized to create a membrane to separate the plastic from the fluids of the inner eye - and this membrane was optically clear. This membrane took the place of the endothelium and remained clear for 20 years. Nature impresses us with a great variety of reactive possibilities in the adaptation of its tissues to new conditions and substances. Sound progress in medicine is easiest when we work along with the physiological currents of beneficial reaction and adaptation. To understand the direction and the limits of nature's reaction is always the first step toward progress in tissue engineering. It is in this sense that the observations here will contribute to progress in the creation of artificial windows in the shell of the eye, with the aim of maintaining and possibly also of correcting vision.

\section{References}

Aquavella JV, Rao GN, Brown AC, Harris JK (1982) Keratoprosthesis. Results, complications, and management. Ophthalmo$\log y 89: 655-660$

Barnham JJ, Roper-Hall MJ (1983) Keratoprosthesis: a long term review. Br J Ophthalmol 67:468-474

Cordona $H$ (1962) Keratoprosthesis, acrylic optical cylinder with supporting interlamellar plate. Am J Ophthalmol 54:284-294

Cordona H (1969) Mushroom transcorneal keratoprosthesis (nut and bolt). Am J Ophthalmol 68:604-612

Cordona H, Castroviejo R, De Voe GA (1962) The Cordona keratoprostesis: flirt clinical evaluation. XIX Concilium Ophthalmol $11: 1211-1229$

Green WR (1984) Discussion paper \#10.1984 Meeting, Am Ophthalmol Soc

Rizzuti AB (1983) Corneal damage: keratoprosthesis considered final opportunity for restored vision. Ophthalmol Times $8: 1$ and 53

Wolter JR (1982a) Lens implant cytology. Ophthalmic Surg 13:939-942

Wolter JR (1982 b) Cell life on the surface of lens implants. Graefe's Arch Clin Exp Ophthalmol 218:244-249

Wolter JR (1983a) Morphology of the capsule-like portion of the reactive membranes on intraocular lens implants. Graefe's Arch Clin Exp Ophthalmol 220:58-65

Wolter JR (1983b) Fusion of macrophages on lens implants resulting in the formation of giant cells. Graefe's Arch Clin Exp Ophthalmol $221: 1-7$

Wolter JR (1984) Cytopathology of intraocular lens implantation. Ophthalmology (in press)

Wolter JR, Lichter PR (1983) Fibroblast-like cells on intraocular lens implants phagocytosing erythrocytes. Br J Ophthalmol 67:641-645

Wolter JR, Sugar (1984) Reactive cellular membrane on a piece of glass: removed after two years in the anterior chamber. Am Intra-Ocular Implant Soc $\mathbf{J}$ (in press)

Received June 18, 1984 